\title{
Reverse Vaccinology: Developing Vaccine Against MDR Acinetobacter baumannii
}

\author{
Ravinder Singh ${ }^{1}$, Neena Capalash ${ }^{2}$ and Prince Sharma ${ }^{1 *}$ \\ ${ }^{1}$ Department of Microbiology, Panjab University, Chandigarh, India \\ ${ }^{2}$ Department of Biotechnology, Panjab University, Chandigarh, India
}

*Corresponding author: Prince Sharma, Department of Microbiology, Panjab University, Chandigarh 160014, India, Tel: 91-9815957903; E-mail: princess@pu.ac.in

Received date: May 09, 2016; Accepted date: May 20, 2016; Published date: May 24, 2016

Copyright: (c) 2016 Singh R, et al. This is an open-access article distributed under the terms of the Creative Commons Attribution License, which permits unrestricted use, distribution, and reproduction in any medium, provided the original author and source are credited.

\begin{abstract}
Emergence of resistance against drugs viz. carbapenems, fluoroquinolones, aminoglycosides is the major problem associated with Acinetobacter baumannii infections thus making it imperative to develop a suitable vaccine as an effective treatment option. Previous studies reveal that outer membrane proteins of $A$. baumannii could serve as vaccine candidates and provide partial or complete immunity against lethal doses in various mouse models. Recently, we have shown reverse vaccinology as a powerful tool to identify vaccine candidates. The immunoprotective efficacy of an outer membrane, putative pilus assembly protein, FilF, identified as a potential vaccine candidate was validated in $A$. baumannii associated murine pneumonia model and was found to provide $50 \%$ survival against $A$. baumannii lethal doses. NucAb, an outer membrane nuclease identified in silico as $A$. baumannii vaccine candidate, provided $20 \%$ survival on active and $40 \%$ survival on passive immunization.
\end{abstract}

\section{Short Communication}

Acinetobacter baumannii is a fast emerging nosocomial pathogen that has gained attention of medical fraternity worldwide $[1,2]$. It infects the patients in hospitals or immunocompromised individuals and makes the treatment difficult due to its multidrug resistance nature [3]. All commonly available antibiotics such as carbapenems, fluoroquinolones and aminoglycosides have failed to control this pathogen [4-7]. Colistin, considered as the last resort of antibiotic treatment, is now used against this pathogen but there are reports of emergence of colistin resistant $A$. baumannii strains [8-10]. Therefore, other than antibiotics, vaccine development appears as the promising and effective treatment option. People in intensive care units or in hospitals for longer durations can be vaccinated prophylactically before admission in order to protect them from this nosocomial pathogen, besides the therapeutic use of vaccine.

Currently, vast information about genomes and proteomes of $A$. baumannii strains is available and promising vaccine candidates can be identified using the computational tools [11-15]. These candidates can further be validated in laboratories using animal models. Highly immunogenic antigens showing little variation among different strains can be determined by conventional techniques but they are time consuming and can be applied only for cultivable microorganisms. Reverse Vaccinology allows a rapid and efficient analysis to find putative immunogenic antigens. This approach can be incorporated in initial study (Figure 1) and can provide such novel proteins which could not be found by conventional vaccinology [16-19]. From the available proteome of pathogen, one can predict putative antigens associated with surface as ideal vaccine candidates. Once shortlisted, these candidates can be cloned and overexpressed in E. coli and purified by affinity chromatography. Their immunogenicity can be validated in vivo in suitable animal models.

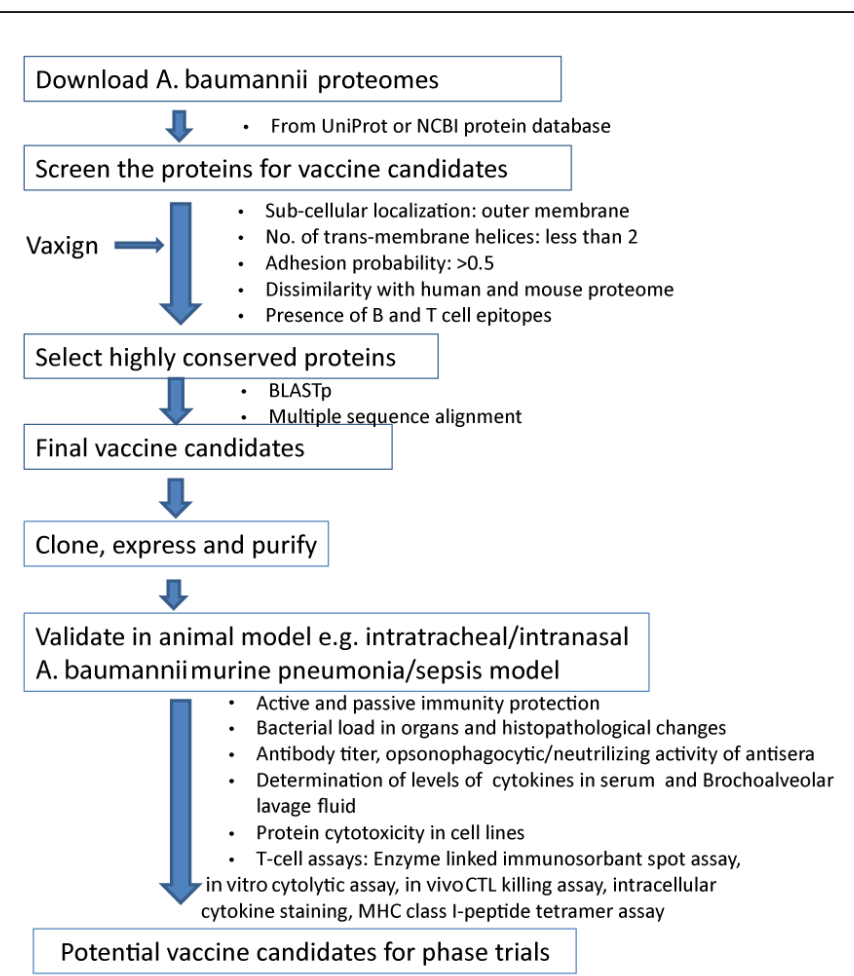

Figure 1: Experimental design for the prediction and validation of immunoprotective efficacy of vaccine candidates from $A$. baumannii proteome.

This approach has successfully led to identification of antigens capable of eliciting protective immunity against $N$. meningitidis group B [15]. Similarly, conserved surface proteins conferring cross- 
protective immunity in several pathogens such as Porphyromonas gingivalis [12], Streptococcus pneumoniae [19], Chlamydia pneumoniae [20] and Streptococcus agalactiae [21] have been identified by reverse vaccinology and successfully used. In our earlier published work [22,23], we have analyzed the proteomes of $A$. baumannii strains and predicted 51 vaccine candidates and validated the immunoprotective potential of two of them (an outer membrane nuclease, NucAb, and FilF, a putative pilus assembly protein) in murine pneumonia model.

FilF is a putative pilus assembly protein that fulfilled in silico the criteria of an ideal vaccine candidate [22]. Its localization in outer membrane makes it interact with host immune system during infection. It has no trans-membrane helix that alleviates the problems encountered in cloning of this protein. And most importantly, its complete dissimilarity with the human and mouse proteome which does not leave any chance of autoimmune response generation by the host. Several other properties of this protein such as high adhesion probability, number of B cell and MHC class I \& II binding epitopes and its ability to bind to MHC molecules strongly make it a promising vaccine candidate. The exact role of FilF in $A$. baumannii is unknown and no information is available how it was named as FilF. Conservation analysis by BLAST and Multalin shows its exclusive presence in genus Acinetobacter with little variation. Nuclease or NucAb, another vaccine candidate identified, was also found to be highly conserved in $A$. baumannii strains and several virulent Acinetobacter species [23].

To study the vaccine potential of candidates, there are a number of animal models developed. Mostly, mouse sepsis models are used to check the bacterial load in various organs and mice survival after lethal dose inoculation [24-27]. Pneumonia models by intranasal route have also been used in several studies [28-32] but this model does not aid in the survival parameter studies as intranasal route does not cause death of mice due to massive clearance of bacteria. Therefore, intranasal route has been used to cause pneumonia and bacterial load determination in lungs followed by intraperitoneal route to cause sepsis to determine the survival rate in mice [31]. In our studies [22, 23], we developed $A$. baumannii associated intratracheal murine pneumonia model by standardizing $A$. baumannii dose to cause lethal infection in $\mathrm{BALB} / \mathrm{c}$ female mice. In our intranasal route attempts, it was found that mice clear a large proportion of bacterial dose and infection was not established in the lungs. Also, intranasal route with higher doses such as $10^{8}$ and $10^{9} \mathrm{cfu}$ was unable to cause the death of mouse observed for a week. Our motive was to develop a pneumonia model which not only provides the bacterial load in lungs studies but also the survival of immunized mice. Therefore, we used intratracheal route to install live bacteria directly into the trachea leading to the infection (lobar pneumonia) establishment. A. baumannii dose was standardized and it was found that $10^{8} \mathrm{cfu}$ administered intratracheally caused the mice death within 24 to $48 \mathrm{hr}$. Hence, an $A$. baumannii associated murine pneumonia model was developed and immunoprotective efficacy of FilF was investigated in the lab. Several parameters such as antibody (IgG) titer and their neutralizing activity, bacterial burden in lungs, histopathological analysis, serum cytokine levels and survival rate were determined in this mouse model [22].

Decrease in bacterial load due to immunization is the indicator of vaccine efficacy. Bacteria spread to host organs and multiply leading to death. Immunization results in reduction in bacterial load and hence increased survival rate of host [22-24,26-28,31,32].
Cytokines perform various functions in host cell and are associated with the infection severity and inflammation. They are released by the infected cell to activate and recruit the immune cells against the pathogen. Levels of pro- as well as anti-inflammatory cytokines are determined to evaluate the efficacy of vaccines. Immunization with vaccine candidates decreases the levels of pro-inflammatory and increases the levels of anti-inflammatory cytokines [26,31]. Epithelial and endothelial cells release IL-33 on invasion by pathogen and on pro-inflammatory stimulation. IL-33 is an inducer of Th2 immune responses. It strongly promotes Th2 cytokine production and aids the pathogenesis of Th2 related diseases, particularly asthma [33]. IL-6 performs as pro- as well as anti-inflammatory cytokine and its levels are elevated in inflammatory states [34]. TNF- $\alpha$ and IFN- $\gamma$ are released during infection and are associated with the systemic inflammation. IL- $1 \beta$ is another pro-inflammatory cytokine that is associated with the innate immunity and host responses on bacterial infection [35]. We have shown that FilF immunization significantly reduced the levels of pro-inflammatory serum cytokine such as TNF- $\alpha$, IFN- $\gamma$, IL- $1 \beta$, IL-33 and IL-6 whereas IL-10 (anti-inflammatory) levels remained comparable in unimmunized and immunized mice [22]. Previous reports have also demonstrated the effect of immunization on decrease in the levels of pro-inflammatory cytokines [24,31,32]. These reductions in cytokine levels are supported by the histopathology studies in which clear difference in infection severity between the organs of unimmunized and immunized mice can be seen $[22,23,31,32]$.

In our studies [22,23], we found that immunization with outer membrane proteins elicited high antibody titer but was unable to provide $100 \%$ protection against lethal bacterial dose. There could be several possible reasons for this such as down regulation of certain membrane proteins by bacteria during the acquisition of antibiotic resistance and during the immunity development [36]. Several lines of evidence in our published study [22,23] supported the fact that FilF as sub-unit vaccine could limit the infection and provided protection (50\% survival) from lethal bacterial dose challenge. On the other hand, active immunization with nuclease provided $20 \%$ survival which increased to $40 \%$ after passive immunization. The future of the $A$. baumannii vaccinology should be focused on a better and clear understanding of the natural infection process in both animal models and human beings. The route of infection and bacterial dose are critical in causing the infection. Appropriate mouse models and more promising vaccine candidates can be explored to develop the best way to protect individuals at high risk. Antigens predicted by reverse vaccinology may represent most appropriate vaccine candidates.

\section{Disclosures}

The authors have no conflicts of interest nor any financial interests to disclose.

\section{References}

1. Joly-Guillou ML (2005) Clinical impact and pathogenicity of Acinetobacter. Clin Microbiol Infect 11: 868-873.

2. Peleg AY, Seifert H, Paterson DL (2008) Acinetobacter baumannii: emergence of a successful pathogen. Clin Microbiol Rev 21: 538-582.

3. Fournier PE, Richet H (2006) The epidemiology and control of Acinetobacter baumannii in health care facilities. Clin Infect Dis 42 : 692-699. 
4. Higgins PG, Dammhayn C, Hackel M, Seifert H (2010) Global spread of carbapenem-resistant Acinetobacter baumannii. J Antimicrob Chemother 65: 233-238.

5. Göttig S, Pfeifer Y, Wichelhaus TA, Zacharowski K, Bingold T, et al. (2010) Global spread of New Delhi metallo- $\hat{I}^{2}$-lactamase 1. Lancet Infect Dis 10: 828-829.

6. Rodríguez-Martínez JM, Nordmann P, Ronco E, Poirel L (2010) Extended-spectrum cephalosporinase in Acinetobacter baumannii. Antimicrob Agents Chemother 54: 3484-3488.

7. Doi Y, Husain S, Potoski BA, McCurry KR, Paterson DL (2009) Extensively drug-resistant Acinetobacter baumannii. Emerg Infect Dis 15: 980-982.

8. Taneja N, Singh G, Singh M, Sharma M (2011) Emergence of tigecycline \& colistin resistant Acinetobacter baumanniii in patients with complicated urinary tract infections in north India. Indian J Med Res 133: 681-684.

9. Adams MD, Nickel GC, Bajaksouzian S, Lavender H, Murthy AR, et al. (2009) Resistance to colistin in Acinetobacter baumannii associated with mutations in the PmrAB two-component system. Antimicrob Agents Chemother 53: 3628-3634.

10. Qureshi ZA, Hittle LE, O'Hara JA, Rivera JI, Syed A, et al. (2015) Colistin-resistant Acinetobacter baumannii: beyond carbapenem resistance. Clin Infect Dis 60: 1295-1303.

11. Chiang MH, Sung WC, Lien SP, Chen YZ, Lo AF, et al. (2015) Identification of novel vaccine candidates against Acinetobacter baumannii using reverse vaccinology. Hum Vaccin Immunother 11: 1065-1073.

12. Ross BC, Czajkowski L, Hocking D, Margetts M, Webb E, et al. (2001) Identification of vaccine candidate antigens from a genomic analysis of Porphyromonas gingivalis. Vaccine 19: 4135-4142.

13. Moriel DG, Beatson SA, Wurpel DJ, Lipman J, Nimmo GR, et al.(2013) Identification of novel vaccine candidates against multidrug-resistant Acinetobacter baumannii. PLoS One 8: e77631

14. Singh R (2015) In silico analysis of Burkholderia pseudomallei proteome to predict potential vaccine candidate proteins. Int J Pharm Bio Sci 6: 957-962.

15. Singh R, Garg N, Capalash N, Kumar R, Kumar M, et al. (2014) In silico Analysis of Acinetobacter baumannii Outer Membrane Protein BamA as a Potential Immunogen. Int J Pure Appl Sci Technol 21: 32-39.

16. Rappuoli R (2001) Reverse vaccinology, a genome-based approach to vaccine development. Vaccine 19: 2688-2691.

17. He Y, Xiang Z, Mobley HL (2010) Vaxign: the first web-based vaccine design program for reverse vaccinology and applications for vaccine development. J Biomed Biotechnol 2010: 297505.

18. Pizza M, Scarlato V, Masignani V, Giuliani MM, Aricò B, et al. (2000) Identification of vaccine candidates against serogroup $\mathrm{B}$ meningococcus by whole-genome sequencing. Science 287: 1816-1820.

19. Wizemann TM, Heinrichs JH, Adamou JE, Erwin AL, Kunsch C, et al. (2001) Use of a whole genome approach to identify vaccine molecules affording protection against Streptococcus pneumoniae infection. Infect Immun 69: 1593-1598.

20. Montigiani S, Falugi F, Scarselli M, Finco O, Petracca R, et al. (2002) Genomic approach for analysis of surface proteins in Chlamydia pneumoniae. Infect Immun 70: 368-379.
21. Maione D, Margarit I, Rinaudo CD, Masignani V, Mora M et al. (2005) Identification of a universal Group $B$ streptococcus vaccine by multiple genome screen. Science 309: 148-150.

22. Singh, R, Garg N, Shukla G, Capalash N, Sharma P (2016) Immunoprotective Efficacy of Acinetobacter baumannii Outer Membrane Protein, FilF, Predicted In silico as a Potential Vaccine Candidate. Front Microbiol 7: 158.

23. Garg N, Singh R, Shukla G, Capalash N, Sharma P (2015) Immunoprotective potential of in silico predicted Acinetobacter baumannii outer membrane nuclease, NucAb. Int J Med Microbiol 306: $1-9$.

24. McConnell MJ, Pachon J (2010) Active and passive immunization against Acinetobacter baumannii using an inactivated whole cell vaccine. Vaccine 29: 1-5.

25. McConnell MJ, Dominguez-Herrera J, Smani Y, Lopez-Rojas R, DocoboPerez F, et al. (2010) Vaccination with outer membrane complexes elicits rapid protective immunity to multidrug-resistant Acinetobacter baumannii. Infect Immun 79: 518-526.

26. McConnell MJ, Rumbob C, Boub G, Pachona J (2011) Outer membrane vesicles as an acellular vaccine against Acinetobacter baumannii. Vaccine 29: 5705- 5710.

27. Luo G, Lin L, Ibrahim AS, Baquir B, Pantapalangkoor P, et al. (2012) Active and passive immunization protects against lethal, extreme drugresistant Acinetobacter baumannii infection. PLoS One 7: e29446.

28. Joly-Guillou ML, Wolff M, Pocidalo JJ, Walker F, Carbon C (1997) Use of a new mouse model of Acinetobacter baumannii pneumonia to evaluate the postantibiotic effect of imipenem. Antimicrob Agents Chemother 41: 345-351.

29. Smani Y, Docobo-Perez F, McConnell MJ, Pachon J (2011) Acinetobacter baumannii-induced lung cell death: role of inflammation, oxidative stress and cytosolic calcium. Microb Pathog 50: 224-232.

30. Harris G, Kuo Lee R, Lam CK, Kanzaki G, Patel GB, et al. (2013) A mouse model of Acinetobacter baumannii-associated pneumonia using a clinically isolated hypervirulent strain. Antimicrob Agents Chemother 57: 3601-3613.

31. Huang W, Yao Y, Long Q, Yang X, Sun W, et al. (2014) Immunization against multidrug-resistant Acinetobacter baumannii effectively protects mice in both pneumonia and sepsis models. PLoS One 9: e100727.

32. KuoLee R, Harris G, Yan H, Xu HH, Conlan WJ, et al. (2015) Intranasal immunization protects against Acinetobacter baumannii-associated pneumonia in mice. Vaccine 33: 260-267.

33. Miller AM (2011) Role of IL-33 in inflammation and disease. J Inflamm (Lond) 8: 22.

34. Scheller J, Chalaris A, Schmidt-Arras D, Rose-John S (2011) The pro- and anti-inflammatory properties of the cytokine interleukin-6. Biochim Biophys Acta 1813: 878-888.

35. Eder C (2009) Mechanisms of interleukin-1beta release. Immunobiology 214: 543-553.

36. Vila J, Martí S, Sánchez-Céspedes J (2007) Porins, efflux pumps and multidrug resistance in Acinetobacter baumannii. J Antimicrob Chemother 59: 1210-1215. 